\title{
Estrogen-dependent expression and subcellular localization of the tight junction protein claudin-4 in HEC-1A endometrial cancer cells
}

\author{
MARIA E. CUEVAS ${ }^{1}$, JENNA M. GASKA ${ }^{1}$, ANDREA C. GIST ${ }^{1}$, JONATHAN M. KING ${ }^{2}$, \\ REBECCA A. SHELLER ${ }^{1}$ and MARIA C. TODD ${ }^{1}$ \\ ${ }^{1}$ Biology Department, Southwestern University, Georgetown, TX 78626; \\ ${ }^{2}$ Biology Department, Trinity University, San Antonio, TX 78212, USA
}

Received March 26, 2015; Accepted April 30, 2015

DOI: $10.3892 /$ ijo.2015.3030

\begin{abstract}
Endometrial cancer is the most common female reproductive cancer in the United States and is associated with deregulated tight junction protein expression. Given the highly estrogen-responsive nature of this tissue, we investigated the effects of estrogen and its agonist, 4-OH TAM, on the expression and subcellular localization of the tight junction protein claudin-4 (CLDN-4), in HEC-1A endometrial cancer cells. In untreated HEC-1A cells, we observed dramatic overexpression of claudin-4 protein. In addition, differential detergent extraction analysis indicated that claudin- 4 was localized primarily in the membrane but also found in the cytosolic, nuclear and cytoskeletal fractions. Upon exposure of HEC-1A to estradiol $\left(E_{2}\right)$, we observed a biphasic effect both on the overall expression of claudin-4 protein and on its cytosolic and cytoskeletal presence as demonstrated by immunoblot analysis. Immunofluorescence analysis also revealed a biphasic effect of $E_{2}$ on claudin- 4 expression. In contrast, we observed no changes in expression levels nor in the subcellular distribution patterns of claudin-4 in HEC-1A cells treated with different concentrations of 4-OH TAM. The intracellular presence of CLDN-4 coupled with the biphasic effects of $E_{2}$ on CLDN-4 expression in the cytoskeleton suggest that this protein may be involved in cell signaling to and from TJs.
\end{abstract}

\section{Introduction}

According to the American Cancer Society (1), endometrial cancer is the most common female reproductive cancer in the United States with an incidence of 1 in 37 women. The effects

Correspondence to: Dr Maria E. Cuevas, Biology Department, Southwestern University, 1001 E University Ave., Georgetown, TX 78626, USA

E-mail: cuevasm@southwestern.edu

Key words: estrogen biphasic effect, claudin-4, endometrium of estradiol $\left(\mathrm{E}_{2}\right)$ on reproductive tract structure and function are well known. Recently, however, studies have indicated a role for $E_{2}$ in tumor initiation and progression through its promotion of the proliferative, migratory and invasive capabilities of cells (2-6). Many of the changes that occur in the endometrium during tumorigenesis are similar to those observed during implantation. For example, both processes exhibit diminished endometrial cell to cell attachment through destabilization of tight junctions (TJs), expression of matrix metalloproteinases, differential expression of integrins and angiogenesis (7).

TJs consist of a complex of proteins located on the apical side of cells and are important for regulating paracellular transport and maintaining cell polarity (8). Furthermore, TJs are essential for the tight sealing of cellular sheets necessary to preserve the structural integrity of tissues and organs. Recent studies also suggest a role for TJ proteins in recruiting signaling proteins that regulate processes such as gene transcription, cellular proliferation, differentiation and morphogenesis (8). The TJ protein complex consists of three types of integral membrane proteins; claudins (CLDNs), occludin and junctional adhesion molecules (JAMs). Claudins are the predominant molecular component of TJs and are essential both for their assembly and function $(8,9)$. CLDNs belong to a 24-member protein family that display distinctive tissue-specific expression and are involved in multiple normal cellular processes. In addition, alterations in CLDN gene expression or changes in subcellular localization have been shown to be associated with tumor progression (10).

Specifically, increases in CLDN-3 and -4 expression have been observed in uterine serous papillary carcinoma $(11,12)$, clear-cell endometrial carcinoma (11) and uterine carcinosarcoma (13). Notably, overexpression of CLDN-3 and -4 was associated with a poor clinical outcome (12). Endometrioid adenocarcinomas expressing particularly high levels of claudin-3 and -4 proteins have been found by electron microscopy to exhibit morphologically disrupted TJs (10). Consistent with these findings, overexpression of these two claudin proteins has been positively correlated with tumor progression in the endometrium and increased myometrial invasion (10). In contrast to the overexpression of claudin-3 and -4 observed in endometrial cancer, endometriosis appears 
to be associated with a decrease in the levels of these two proteins $(13,14)$.

The reason for the upregulation of claudin-3 and -4 in certain endometrial tumors is currently unclear but given its role in the physiology of the endometrium, it is possible that $\mathrm{E}_{2}$ may be involved. Whereas two previous studies have shown that exposure of MCF-7 breast cancer cells to low concentrations of $E_{2}$ results in a decrease in claudin- 4 gene expression $(15,16)$, there are very few published studies demonstrating the effects of $\mathrm{E}_{2}$ on claudin expression in endometrial cancer cells. In the current study, therefore, we investigated the effects of varying $E_{2}$ concentrations on the expression and subcellular localization of CLDN-4.

\section{Materials and methods}

Cell lines and tissue culture conditions. The endometrial cancer call line HEC-1A was obtained from ATCC (Manasas, VA, USA) and cultured in McCoys 5A supplemented with $10 \%$ fetal bovine serum (FBS) and $1 \%$ penicillin/streptomycin $/ 2 \mathrm{~mm}$ L-glutamine (PSG) purchased from Life Technologies. Cells were maintained at $37^{\circ} \mathrm{C}$ in a $5 \% \mathrm{CO}_{2}$ atmosphere.

Compounds. The compounds estradiol $\left(\mathrm{E}_{2}\right)$ and 4-hydroxytamoxifen (4-OH TAM) were purchased from Sigma and dissolved in $100 \%$ ethanol, stored, and protected from light in stock solutions of $1 \mathrm{mM}$ at $-20^{\circ} \mathrm{C}$. The final concentration of ethanol in culture media was always $<0.1 \%$ (v/v).

$E_{2}$ and 4-OH tamoxifen exposure experiment. Cells were plated $\left(2 \times 10^{5}\right.$ cells/well) into 6 -well plates or seeded ( $1 \times 10^{6}$ cells) onto $25 \mathrm{~cm}^{2}$ culture flask and cultured with their respective media supplemented with $10 \%$ FBS-1\% PSG for 48 h. Logarithmic phase cells were washed twice with PBS and cells were serum starved for $24 \mathrm{~h}$ before the medium was replaced with $10 \%$ charcoal treated fetal bovine serum (CSFBS) (HyClone) with different concentrations of $E_{2}$ or $4-\mathrm{OH}$ TAM ranging from $0-100 \mathrm{nM}$. The medium was replaced daily to ensure constant hormone concentration. Cells were harvested after $48 \mathrm{~h}$ to prepare whole cells protein extracts or subcellular fractions.

Subcellular fractionation. HEC-1A cells were treated with a series of commercial extraction buffers (Calbiochem) according to manufactures instructions to obtain cytosolic, membranous, nuclear and cytoskeletal fractions.

Western blot analysis. Proteins in whole cell extracts and subcellular fractions were suspended in $4 \mathrm{X}$ sample buffer (40\% v/v glycerol, $4 \%$ SDS, $0.5 \% \mathrm{w} / \mathrm{v}$ bromophenol blue, $10 \% \beta$-mercaptoethanol and 0.16 M Tris, $\mathrm{pH} 7.0$ ), subjected to electrophoresis on precast $12 \%$ SDS-polyacrylamide gels and electrophoretically transferred to Immobilon-P PVDF. The membranes were probed for $1 \mathrm{~h}$ at room temperature with $2 \mu \mathrm{g} / \mathrm{ml}$ rabbit polyclonal claudin $-3,3 \mu \mathrm{g} / \mathrm{ml}$ mouse monoclonal claudin-4 (Life Technologies) or $1 \mu \mathrm{g} / \mathrm{ml}$ of rabbit anti-actin (I-19) (Santa Cruz Biotechnology, Santa Cruz, CA, USA) primary antibodies in 5\% milk/PBS solution. Subsequently, the membranes were incubated with 1:3000 horseradish peroxidase-conjugated secondary antibody (goat anti-rabbit or goat-anti mouse; IgG; Bio-Rad Laboratories) for $1 \mathrm{~h}$ at room temperature. For signal detection the enhanced chemiluminescence ECL-plus kit (Amersham, Buckinghamshire, UK) was used according to manufacturer's instructions.

Confocal microscopy. Untreated or hormone treated log phase cells were harvested and plated at $5 \times 10^{5}$ cells per chambered coverslide (Lab-Tek, Fisher Scientific) and grown at $37^{\circ} \mathrm{C}$ until $80 \%$ confluency. Cells were then rinsed with pre-cooled PBS three times and fixed in pre-cooled $95 \%$ ethanol for $30 \mathrm{~min}$ on ice. Following rehydration in PBS slides were blocked with $3 \%$ BSA, $0.05 \%$ saponin in PBS. Claudin-4 anti-sera (1:250) (Life Technologies) was applied overnight at $4^{\circ} \mathrm{C}$, followed by three wash cycles with PBS-saponin and incubation with goat anti-mouse-IgG conjugated to Rhodamine Red-X (Jackson ImmunoResearch) for $1 \mathrm{~h}$ at room temperature. Nuclei were counterstained with Hoechst 33342 (1:1000) and filamentous actin stained with Alexa 647-Phalloidin (1:500) followed by PBS washing and treatment with Prolong Gold antiFade Reagent (Life Technologies). High-resolution 1024X1024 images were collected using a Nikon A1R confocal system with the 40X Plan Fluor NA 1.4 oil objective. The images were thresholded, normalized and maximum intensity projections from $8 \mu \mathrm{m} \mathrm{z}$-stacks were collected. Fluorescent image intensity was quantified and presented as the mean \pm SEM. Significantly different groups were determined by ANOVA with Tukey's HSD analysis $(\mathrm{p}<0.05)$.

\section{Results}

Claudin-4 expression in normal human tissues and reproductive cancer cell lines. To determine the variation of expression of CLDN-4 in normal tissues, we analyzed a panel of protein extracts derived from human bladder, breast, cervix, kidney, ovary, placenta, prostate, testis and uterus (pre-made tissue western blot purchased from ProSci). As seen in Fig. 1 the strongest expression of CLDN-4 was seen in the placenta, followed by the bladder, cervix and kidney. A very faint signal was observed in prostate and breast tissue. There was no obvious expression of CLDN-4 in the uterus, ovary and testis.

In contrast to the low expression of CLDN-4 in normal uterine tissue (Fig. 1), the endometrial adenocarcinoma cell line HEC-1A, showed robust expression of CLDN-3 and -4 (Fig. 2). A less intense CLDN-4 signal was observed in the endometrial cancer cell line RL95-2. There was no detectable signal in the cancer cell lines HEC-1B (endometrial), HeLa (cervical) and SK-OV-3 (ovarian). Similarly, there were non-detectable to low levels of CLDN-3 in all but the HEC-1A cancer cells. These data indicate that the CLDN-3 and CLDN-4 proteins are abnormally overexpressed in the HEC-1A cell line (Fig. 2). In summary, these data show differential expression patterns of CLDN-3 and -4 between the different cancer cell lines.

Subcellular localization of claudin-4 protein. We used differential detergent cell fractionation, to assess the subcellular localization of CLDN-4 in HEC-1A cells. As shown in Fig. 3, we observed CLDN-4 expression in all four subcellular fractions, cytosolic (C), membranous (M), nuclear $(\mathrm{N})$ and 


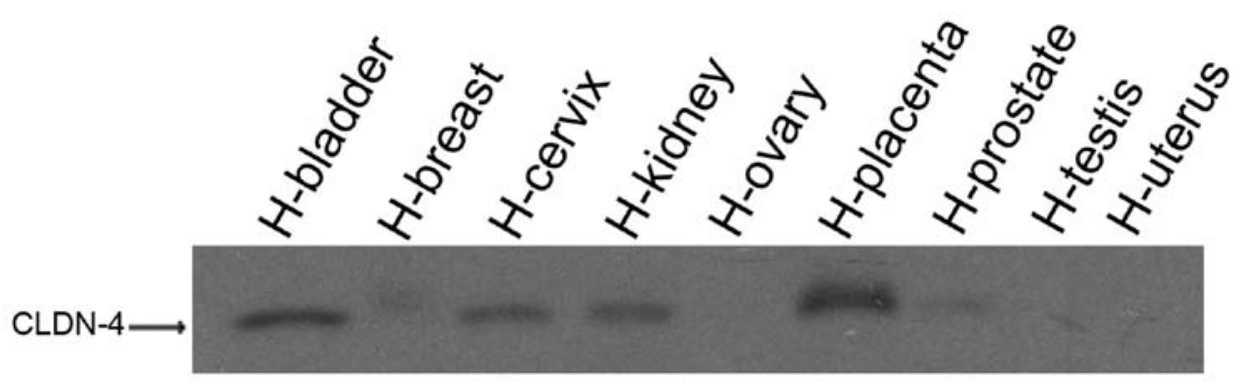

Figure 1. Claudin-4 (CLDN-4) expression in a panel of normal human tissues. A pre-made western blot (ProSci) containing a panel of protein extracts derived from different human tissues was probed with anti-claudin-4. The strongest expression of CLDN-4 was seen in the placenta, followed by bladder, cervix and kidney, A very faint signal was observed in prostate and breast. There was no detectable expression of CLDN-4 in the uterus, ovary and testis.
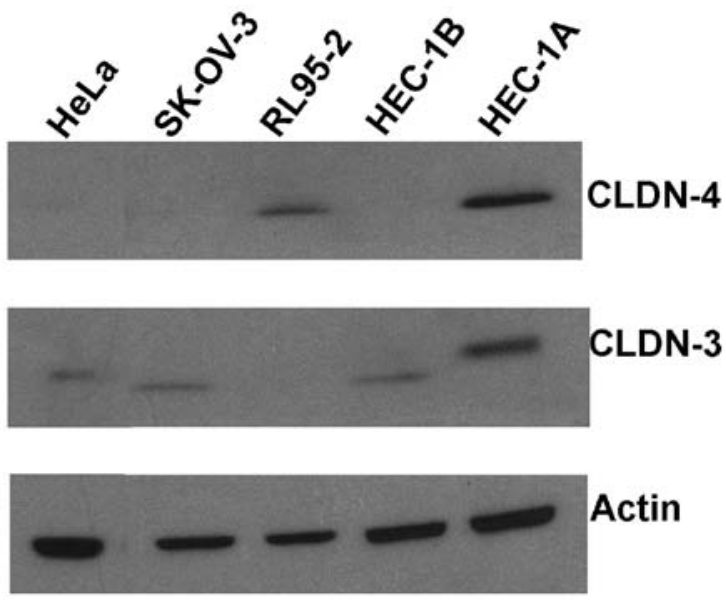

Figure 2. Overexpression of claudin (CLDN)-3 and -4 in the endometrial cancer cell line HEC-1A. A panel of female reproductive cancer cell lines was subjected to immunoblot analysis using antibodies to claudin- $3,-4$ and actin. Overexpression of CLDN-3 and -4 was observed in the endometrial adenocarcinoma cell line HEC-1A relative to cervical (HeLa), ovarian (SK-OV-3) and the endometrial (RL95-2 and HEC-1B) cancer cell lines.

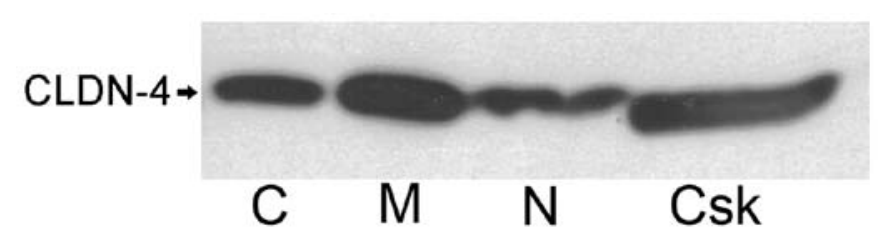

Figure 3. Subcellular localization of claudin-4 (CLDN-4) in HEC-1A cells by differential detergent fractionation. Immunoblots containing subcellular fractions of HEC-1A cells were probed with anti-CLDN-4. The majority of CLDN-4 was localized in the membrane fraction (M) and cytoskeletal (Csk) fractions with readily detectable signal in both the nuclear $(\mathrm{N})$ and cytosolic (C) fractions. The blot is representative of 3 separate experiments.

cytoskeletal (Csk). The most intense signals were in the membranous and cytoskeletal fractions.

Effects of E2 on CLDN-4 expression and subcellular localization. Cells were serum-starved for $24 \mathrm{~h}$ then exposed to $10-100 \mathrm{nM} \mathrm{E}_{2} / \mathrm{CSFBS}$ for $48 \mathrm{~h}$. The medium was replaced daily to ensure constant hormone concentration. As a control, one set of cells was grown in FBS-containing medium. Whole cell protein extracts (Fig. 4A) and subcellular fractions (Fig. 4B) were subsequently analyzed for CLDN-4 expression by immunoblot analysis. As shown in Fig. 4A, $\mathrm{E}_{2}$ effected CLDN-4

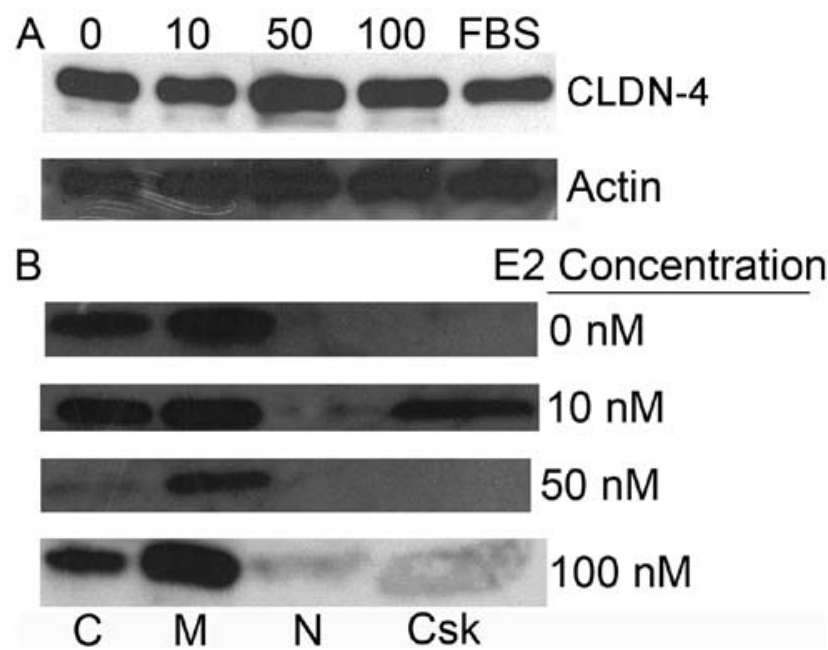

Figure 4. Biphasic effect of estradiol on claudin-4 (CLDN-4) expression in HEC-1A cells. Cells were exposed to a range of estradiol concentrations $(0-100 \mathrm{nM})$ in CSFBS for $72 \mathrm{~h}$. As a control, one set of cells was grown in media containing FBS. Whole cell protein extracts (A) and four cellular subfractions (B) cytosolic (C), membranous (M), nuclear (N), and cytoskeletal (Csk) were subsequently analyzed for CLDN-4 ( $22 \mathrm{kDa})$ expression by immunoblot analyis. Actin was used as a loading control. Estradiol increased CLDN-4 expression with a biphasic effect seen at the two highest concentrations. We observed alterations in the pattern of CLDN-4 subcellular distribution in response to different estradiol concentrations. The blot is representative of 3 separate experiments.

expression in a biphasic manner with the most intense signal observed at $50 \mathrm{nM} \mathrm{E}_{2}$. In addition, we observed alterations in the pattern of CLDN-4 subcellular distribution in response to different $E_{2}$ concentrations. Regardless of the $E_{2}$ concentration, CLDN-4 expression was predominantly observed in the membrane fraction (Fig. 4B). We observed evidence of a biphasic $E_{2}$ effect on both the cytosolic and cytoskeletal presence of CLDN-4 in HEC-1A cells. Specifically, there was a high level of CLDN-4 in cells treated with 0,10 and $100 \mathrm{~nm}$ $\mathrm{E}_{2}$ with barely detectable levels in cells treated with $50 \mathrm{nM}$ $\mathrm{E}_{2}$. Furthermore, high levels of CLDN-4 were observed in the cytoskeletal fractions of cells treated with $10 \mathrm{nM}$ but not in those cells treated with $0 \mathrm{nM}$ and $50 \mathrm{nM} \mathrm{E}_{2}$.

Effects of 4-OH tamoxifen on CLDN-4 expression and subcellular localization. We evaluated the expression of CLDN-4 in response to various 4-OH TAM concentrations (0-100 $\mathrm{nM})$ and found it to be concentration-independent 


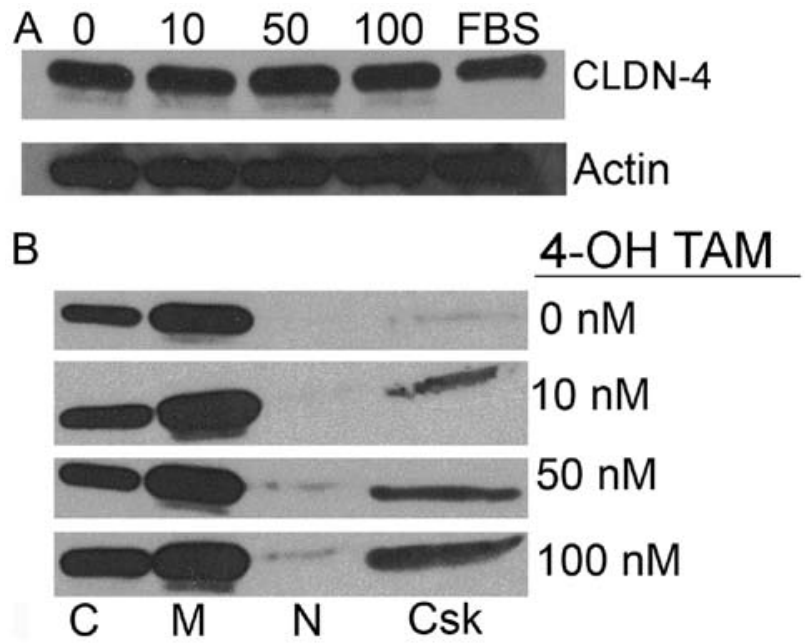

Figure 5. Induction of claudin-4 (CLDN-4) expression by 4-OH tamoxifen (4-OH TAM) in HEC-1A cells. Cells were exposed to a range of 4-OH TAM concentrations $(0-100 \mathrm{nM})$ in CSFBS for $72 \mathrm{~h}$. As a control, one set of cells was grown in media containing FBS. Whole cell protein extracts (A) and four cellular subfractions (B) cytosolic (C), membranous (M), nuclear (N) and cytoskeletal (Csk) were subsequently analyzed for CLDN-4 ( 22 kDa) expression on immunoblots. Actin was used as a loading control. Non-dose dependent induction of claudin-4 expression by 4-OH TAM was observed. However, the effect was not biphasic in nature. No dramatic alterations in the pattern of CLDN-4 subcellular distribution were observed in response to different 4-OH TAM concentrations with the exception of a modest dosedependent increase in signal in the nuclear fraction. Blot is representative of 3 separate experiments.

(Fig. 5A). Similarly, the subcellular distribution pattern of CLDN-4 was also 4-OH TAM-independent. Specifically, we observed the most intense CLDN-4 signal in the membranous fraction closely followed by the cytosolic fraction (Fig. 5B). Readily detectable bands were also observed in the cytoskeletal fraction of the cells exposed to 10-100 nM 4-OH TAM. However, only barely detectable levels of CLDN-4 were observed in the nuclear fractions at all concentrations.

Effects of E2 on CLDN-4 expression and localization by immunofluorescence. CLDN-4 localization was evaluated by indirect immunofluorescence using laser scanning confocal microscopy. $\mathrm{E}_{2}$ supplementation (Fig. 6A) enhanced CLDN-4 expression and localization at cell-cell contacts. HEC-1A cells cultured in the absence of $\mathrm{E}_{2}$ expressed modest levels of CLDN-4 with localization distributed between the cytoplasm and membrane. $\mathrm{E}_{2}(10 \mathrm{nM})$ resulted in a shift toward membrane localization with a slight elevation in expression. Robust elevation of CLDN-4 signal occurred with $50 \mathrm{nM} \mathrm{E}$ supplementation as indicated by clearly delineated cell-cell contacts and a marked elevation in intensity. CLDN-4 signal was apparent in HEC-1A cells treated with $100 \mathrm{nM} \mathrm{E}_{2}$ but the junctional intensity was diminished and intracellular signal more frequent. Quantitation of CLDN-4 fluorescent intensity is presented in Fig. 6B with significant differences found between each $\mathrm{E}_{2}$ treatment group. HEC-1A cells cultured in defined media with FBS exhibited CLDN-4 specific staining at the perijunctional actin ring (Fig. 6C). The junctional intensity of CLDN-4 from cells cultured in media with FBS was statistically undistinguishable from cells cultured in $100 \mathrm{nM}$ $\mathrm{E}_{2}$ (Fig. 6B).

\section{Discussion}

There is a growing body of evidence suggesting that alterations in CLDN expression may be involved in the progression of some cancers (17) such as endometrial carcinoma (11). However, the regulation of these changes in expression are not well understood. Thus, the current study sought to investigate the potential role of $\mathrm{E}_{2}$ and the chemotherapeutic drug, 4-OH TAM, on CLDN-4 expression in the endometrial cancer cell line HEC-1A.

Our findings show that whereas CLDN-4 is either not expressed or barely expressed in the endometrial cell lines HEC-1B and RL95-2, respectively, it is dramatically overexpressed in HEC-1A cancer cells. Furthermore, CLDN-3 was also overexpressed in HEC-1A relative to the other two endometrial cancer cell lines. Notably, there was no detectable CLDN-4 expression in the normal uterine tissue. These findings are consistent with previous studies that have demonstrated elevated CLDN-4 expression with increased endometrial tumor grade (10-12). In addition, the observed lack of CLDN-4 expression in normal uterine tissue agrees with previous studies that demonstrated absent or weak CLDN-4 expression in normal endometrial cells (NEC), proliferative and secretory endometrial tissue $(10,11)$.

Currently, the regulation of CLDN-4 expression in endometrial cells is not well understood. Owing to its major role in the endometrium, we investigated the possible effects of $\mathrm{E}_{2}$ and 4-OH TAM, a known $\mathrm{E}_{2}$ partial agonist in the endometrium, on the expression of CLDN-4. Notably, we observed a clear biphasic effect of $\mathrm{E}_{2}$ on CLDN-4 expression. The lowest levels of expression were seen at $10 \mathrm{nM}$ and $100 \mathrm{nM} \mathrm{E}_{2}$ whereas the level of CLDN-4 expression increased following exposure to $50 \mathrm{nM} \mathrm{E}_{2}$ as demonstrated by both immunoblot and immunofluorescent analyses. Similar to our findings, Gadal et al (16) also observed a decrease in CLDN-4 gene expression in MCF-7 breast cancer cells upon exposure to $10 \mathrm{nM} \mathrm{E}_{2}$. In contrast, Someya et al (18) showed a dose-dependent increase in CLDN-4 protein expression in the Sawano uterine cancer cell line with the highest levels of expression observed at $100 \mu \mathrm{M}$. It should be noted, however, that the concentrations of $\mathrm{E}_{2}$ used in the latter study are above the normal physiological range.

Whereas we and others have shown a biphasic effect of $E_{2}$ in endometrial and breast cancer cells, Zeng et al (19) did not observe an $\mathrm{E}_{2}$ biphasic effect on CLDN-4 expression in human cervical cells. This discrepancy is likely attributable to inherent differences between the two tissue types studied. In addition to CLDN-4, $E_{2}$ has been shown to have a biphasic effect on the levels of another tight junction protein, occludin, in both human vascular epithelial cells (20) and cervical cells (19).

As a complement to the above $\mathrm{E}_{2}$ exposure studies we treated the HEC-1A cells with the endometrial estrogen agonist, 4-OH TAM. Decreased CLDN-4 expression occurred only at concentrations of $100 \mathrm{nM} 4-\mathrm{OH}$ TAM. Owing to the differential effects of 4-OH TAM on endometrial and breast tissues, it is not surprising that Gadal et al (16) observed an increase in CLDN-4 gene expression following treatment of MCF-7 breast cancer cells with 100 nM 4-OH TAM.

We next determined the effect of $\mathrm{E}_{2}$ on the subcellular localization of CLDN-4 in HEC-1A cells. Using differential 

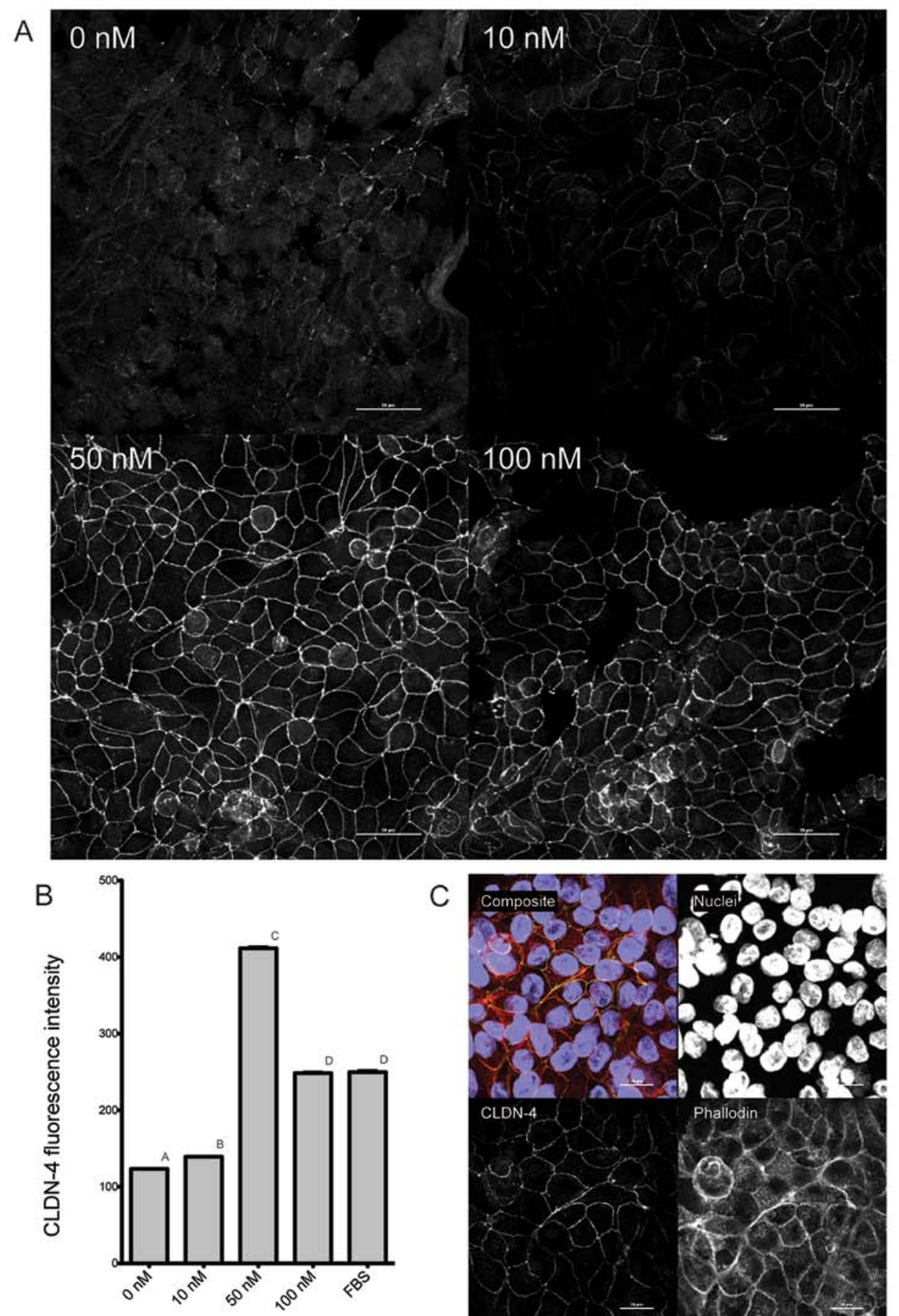

C

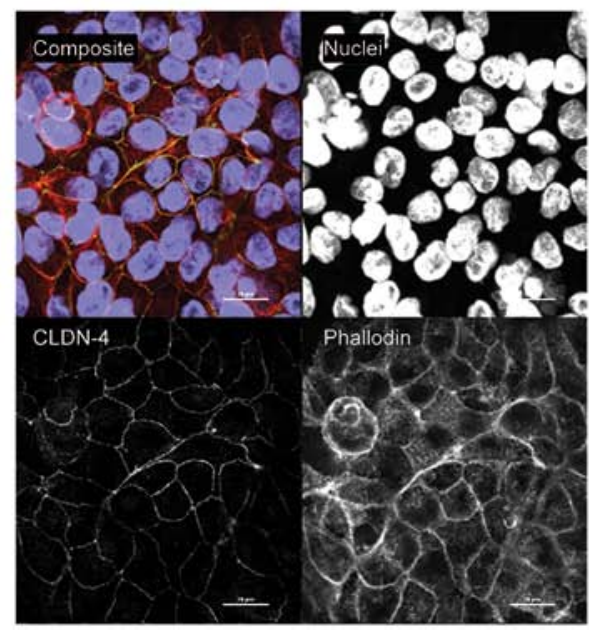

Figure 6. $\mathrm{E}_{2}$-induced intracellular redistribution of claudin-4 (CLDN-4) in HEC-1A cells. Cells were cultured onto chambered coverslides and treated with 0-100 $\mathrm{nM} \mathrm{E}_{2}$. Representative maximum intensity projection images are presented in (A) following CLDN-4 indirect immunofluorescence. Fluorescent image intensity was quantified and presented as the mean \pm SEM (B). Letters indicate significantly different groups as determined by ANOVA with Tukey's HSD analysis ( $\mathrm{p}<0.05)$. (C) Representation of HEC-1A cells cultured in media with FBS. A composite image is presented in addition to the individual channels. Junctional CLDN-4 corresponds to perijunctional F-actin localization. Nuclei were counterstained with Hoechst 33342 and filamentous actin was labeled with Alexa 647 phallodin. Scale bar, $50 \mu \mathrm{m}$.

detergent extraction analysis, we observed CLDN-4 in all four subcellular fractions, membranous, cytosolic, nuclear and cytoskeletal. Specifically, high levels of CLDN-4 were observed in the nuclear fraction at the highest $\mathrm{E}_{2}$ concentration $(100 \mathrm{nM})$. This contrasts with the barely detectable nuclear fraction-specific signal at all other concentrations (0-50 nM). In addition, we observed a biphasic effect of $E_{2}$ on CLDN-4 expression in the cytoskeletal fraction of HEC-1A cells. Immunofluorescence analysis also showed a biphasic effect of $E_{2}$ on the expression of claudin- 4 with a shift to an intracellular localization of claudin- 4 with increasing $E_{2}$ concentration.

Whereas the expression of CLDN-4 in the membrane is expected as a part of its role in the TJ, the significance of the intracellular localization is not clear. Previous studies have reported delocalization of CLDN proteins. For example, Zhu et al (20) observed the presence of CLDN-1, -3, and -4 in the cytoplasm of cells from ovarian epithelial tumors by 
immunofluorescence analysis. Furthermore, Leotlela et al (21) found that CLDN-1 was expressed almost exclusively in the nucleus of benign nevi, or birthmarks, whereas it was located in the cytosolic and membranous fractions in highly metastastic melanoma cells. Lejeune et al (22) showed that the shift of CLDN-4 from the membrane to the cytoskeleton upon exposure of T84 human colonic cells to the host inflammatory mediator prostaglandin $\mathrm{E}_{2}$ correlates with dissociation of CLDN-4 from the TJ and is possibly responsible for the rapid changes in TER that they subsequently observed.

Phosphorylation of CLDN proteins also appears to play a role in their subcellular localization. D'Souza et al (23) demonstrated that the phosphorylation of CLDN-3 on a threonine residue alters the localization of this protein within the membrane and the cytoplasm of ovarian OVCA433 cancer cells. Similarly, phosphorylation of CLDN-4 in HT29 colorectal adenocarcinoma cells weakens the association between CLDN-4 and ZO-1, leading to an increased presence of CLDN-4 in the cytoplasm (24). Furthermore, research has shown that phosphorylation of CLDN-1 in human melanoma cells can result in the redistribution of CLDN-1 to the nucleus or cytoplasm (25).

Our findings underscore the dynamic nature of the TJ as evidenced by the changes in subcellular localization of CLDN-4 upon exposure to $\mathrm{E}_{2}$. Just how these changes in subcellular localization come about is unclear. However, it has been shown that CLDNs can be removed from the plasma membrane by endocytosis into cytoplasmic vesicles (26) and they have been found in extracellular exosomes of cancer tissues (27). However, endocytosis and exocytosis do not explain how an integral membrane protein, with four hydrophobic (27) domains, can dissolve in the cytosol and translocate to the nucleus despite the absence of a nuclear localization sequence (28). The delocalization of CLDNs may indicate a role for CLDN proteins in cell signaling pathways with the PDZ domain of CLDNs providing a promising site for the formation of signaling complexes (29).

Due to their frequent overexpression in numerous cancers and function as receptors for Clostridium perfringens enterotoxin (CPE), CLDN-3 and -4 have been considered as useful targets in treating tumors overexpressing one or both proteins, such as uterine serous papillary carcinoma (30). To prevent wide-spread cytolysis by use of this therapy, researchers have focused on a non-cytotoxic C-terminal fragment of CPE to specifically bind CLDN-3 or -4 , altering the $\mathrm{TJ}$, and subsequently allowing for better drug absorption by affected cells (31). Furthermore, the changes in CLDN-3 and -4 expression in certain cancers have suggested that these proteins may be potential prognostic markers. Indeed, a recent study developed a so-called CURIO score based on CLDN-4 and E-cadherin expression in breast cancer. This score has proven generally accurate in predicting a poorer prognosis for those patients whose breast tumors overexpress these two proteins (32).

\section{Acknowledgements}

This work was supported by the National Science Foundation Major Research Instrumentation (NSF-MRI) Grant (0922258) awarded to M.C.T. and M.E.C., NSF-MRI Grant (1229702) awarded to J.M.K., Joe and Jessie Crump Fund at JP Morgan Bank, the ACS Andrew W. Mellon Integrated Scholarly Grant awarded to M.C.T., M.E.C. and R.A.S., the Howard Hughes Medical Institute through the Undergraduate Science Education Program (52007558), a Sam Taylor Fellowship and the Southwestern University Faculty-Student Collaborative Projects fund awarded to M.E.C. The authors would like to thank Taylor Vickers for his help with the figures.

\section{References}

1. American Cancer Society (online). Available: http://www. cancer.org/cancer/endometrialcancer/detailedguide/endometrialuterine-cancer-key-statistics (updated Feb 3, 2014).

2. Yan Y, Liu H, Wen H, Jiang X, Cao X, Zhang G and Liu G: The novel estrogen receptor GPER regulates the migration and invasion of ovarian cancer cells. Mol Cell Biochem 378: 1-7, 2013.

3. Tsai CL, Wu HM, Lin CY, Lin YJ, Chao A, Wang TH, Hsueh S, Lai $\mathrm{CH}$ and Wang HS: Estradiol and tamoxifen induce cell migration through GPR30 and activation of focal adhesion kinase (FAK) in endometrial cancers with low or without nuclear estrogen receptor $\alpha(E R \alpha)$. PLoS One 8: e72999, 2013.

4. Flamini MI, Sanchez AM, Goglia L, Tosi V, Genazzani AR and Simoncini T: Differential actions of estrogen and SERMs in regulation of the actin cytoskeleton of endometrial cells. Mol Hum Reprod 15: 675-685, 2009.

5. Gentilini D, Busacca M, Di Francesco S, Vignali M, Viganò $P$ and Di Blasio AM: PI3K/Akt and ERK1/2 signalling pathways are involved in endometrial cell migration induced by 17 betaestradiol and growth factors. Mol Hum Reprod 13: 317-322, 2007.

6. Acconcia F, Barnes CJ and Kumar R: Estrogen and tamoxifen induce cytoskeletal remodeling and migration in endometrial cancer cells. Endocrinology 147: 1203-1212, 2006.

7. Tabibzadeh S and Babaknia A: The signals and molecular pathways involved in implantation, a symbiotic interaction between blastocyst and endometrium involving adhesion and tissue invasion. Hum Reprod 10: 1579-1602, 1995.

8. Turksen K and Troy TC: Barriers built on claudins. J Cell Sci 117: 2435-2447, 2004.

9. Schneeberger EE and Lynch RD: The tight junction: A multifunctional complex. Am J Physiol Cell Physiol 286: C1213-C1228, 2004.

10. Pan XY, Wang B, Che YC, Weng ZP, Dai HY and Peng W: Expression of claudin-3 and clauding-4 in normal, hyperplastic, and malignant endometrial tissue. Int $\mathbf{J}$ Gynecol Cancer 17: 233-241, 2007.

11. Santin AD, Bellone S, Siegel ER, McKenney JK, Thomas M, Roman JJ, Burnett A, Tognon G, Bandiera E and Pecorelli S: Overexpression of Clostridium perfringens enterotoxin receptors claudin-3 and claudin- 4 in uterine carcinosarcomas. Clin Cancer Res 13: 3339-3346, 2007.

12. Konecny GE, Agarwal R, Keeney GA, Winterhoff B, Jones MB Mariani A, Riehle D, Neuper C, Dowdy SC, Wang HJ, et al: Claudin-3 and claudin-4 expression in serous papillary, clearcell, and endometrioid endometrial cancer. Gynecol Oncol 109: 263-269, 2008.

13. Gaetje R, Holtrich U, Engels K, Kissler S, Rody A, Karn T and Kaufmann M: Differential expression of claudins in human endometrium and endometriosis. Gynecol Endocrinol 24: 442-449, 2008.

14. Pan XY, Li X, Weng ZP and Wang B: Altered expression of claudin-3 and claudin-4 in ectopic endometrium of women with endometriosis. Fertil Steril 91: 1692-1699, 2009.

15. Seth P, Porter D, Lahti-Domenici J, Geng Y, Richardson A and Polyak K: Cellular and molecular targets of estrogen in normal human breast tissue. Cancer Res 62: 4540-4544, 2002.

16. Gadal F, Starzec A, Bozic C, Pillot-Brochet C, Malinge S, Ozanne V, Vicenzi J, Buffat L, Perret G, Iris F, et al: Integrative analysis of gene expression patterns predicts specific modulations of defined cell functions by estrogen and tamoxifen in MCF7 breast cancer cells. J Mol Endocrinol 34: 61-75, 2005.

17. Morin PJ: Claudin proteins in human cancer: Promising new targets for diagnosis and therapy. Cancer Res 65: 9603-9606, 2005 . 
18. Someya M, Kojima T, Ogawa M, Ninomiya T, Nomura $K$, Takasawa A, Murata M, Tanaka S, Saito T and Sawada N: Regulation of tight junctions by sex hormones in normal human endometrial epithelial cells and uterus cancer cell line Sawano. Cell Tissue Res 354: 481-494, 2013.

19. Zeng R, Li X and Gorodeski GI: Estrogen abrogates transcervical tight junctional resistance by acceleration of occluding modulation. J Clin Endocrinol Metab 89: 5145-5155, 2004.

20. Zhu Y, Brännström M, Janson PO and Sundfeldt K: Differences in expression patterns of the tight junction proteins, claudin 1 , 3,4 and 5, in human ovarian surface epithelium as compared to epithelia in inclusion cysts and epithelial ovarian tumours. Int J Cancer 118: 1884-1891, 2006.

21. Leotlela PD, Wade MS, Duray PH, Rhode MJ, Brown HF, Rosenthal DT, Dissanayake SK, Earley R, Indig FE, Nickoloff BJ, et al: Claudin-1 overexpression in melanoma is regulated by $\mathrm{PKC}$ and contributes to melanoma cell motility. Oncogene 26 3846-3856, 2007.

22. Lejeune M, Moreau F and Chadee K: Prostaglandin E2 produced by Entamoeba histolytica signals via EP4 receptor and alters claudin-4 to increase ion permeability of tight junctions. Am J Pathol 179: 807-818, 2011.

23. D'Souza T, Indig FE and Morin PJ: Phosphorylation of claudin-4 by PKCepsilon regulates tight junction barrier function in ovarian cancer cells. Exp Cell Res 313: 3364-3375, 2007.

24. Tanaka M, Kamata R and Sakai R: EphA2 phosphorylates the cytoplasmic tail of Claudin-4 and mediates paracellular permeability. J Biol Chem 280: 42375-42382, 2005.

25. French AD, Fiori JL, Camilli TC, Leotlela PD, O'Connell MP, Frank BP, Subaran S, Indig FE, Taub DD and Weeraratna AT: PKC and PKA phosphorylation affect the subcellular localization of claudin-1 in melanoma cells. Int J Med Sci 6: 93-101, 2009.
26. Matsuda M, Kubo A, Furuse M and Tsukita S: A peculiar internalization of claudins, tight junction-specific adhesion molecules, during the intercellular movement of epithelial cells. J Cell Sci 117: 1247-1257, 2004.

27. Li J, Sherman-Baust CA, Tsai-Turton M, Bristow RE, Roden RB and Morin PJ: Claudin-containing exosomes in the peripheral circulation of women with ovarian cancer. BMC Cancer 9: 244, 2009.

28. Dhawan P, Singh AB, Deane NG, No Y, Shiou SR, Schmidt C, Neff J, Washington MK and Beauchamp RD: Claudin-1 regulates cellular transformation and metastatic behavior in colon cancer. J Clin Invest 115: 1765-1776, 2005.

29. Heiskala M, Peterson PA and Yang Y: The roles of claudin superfamily proteins in paracellular transport. Traffic 2: 93-98, 2001.

30. Santin AD, Bellone S, Marizzoni M, Palmieri M, Siegel ER, McKenney JK, Hennings L, Comper F, Bandiera E and Pecorelli S: Overexpression of claudin-3 and claudin-4 receptors in uterine serous papillary carcinoma: novel targets for a typespecific therapy using Clostridium perfringens enterotoxin (CPE). Cancer 109: 1312-1322, 2007.

31. Veshnyakova A, Protze J, Rossa J, Blasig IE, Krause G and Piontek J: On the interaction of Clostridium perfringens enterotoxin with claudins. Toxins (Basel) 2: 1336-1356, 2010.

32. Szasz AM, Nemeth Z, Gyorffy B, Micsinai M, Krenacs T, Baranyai Z, Harsanyi L, Kiss A, Schaff Z, Tökés AM, et al: Identification of a claudin-4 and E-cadherin score to predict prognosis in breast cancer. Cancer Sci 102: 2248-2254, 2011. 